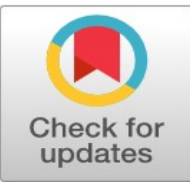

\title{
Dentin microtensile bond strength of 10-MDP contained dental adhesives used in class $V$ cavity
}

\author{
Suttinart Chomcheoy ${ }^{1 *}$, Chayada Teanchai ${ }^{2}$, Apiwat Riddhabhaya ${ }^{3}$ \\ ${ }^{1}$ Department of Dental Public Health, Sirindhorn College of Public Health, Bang Pla Soi, Thailand \\ ${ }^{2}$ Faculty of Dentistry, Mahidol University, Salaya, Thailand \\ ${ }^{3}$ Faculty of Dentistry, University of Western Ontario, London, Canada
}

\author{
Key Words: \\ Microtensile \\ Dental adhesives \\ Class V cavity
}

Received: 16 May 2017

Accepted: 23 August 2017

Published: 16 October 2017

\begin{abstract}
The objective of this study is to evaluate the dentin microtensile bond strength of total etching and self-etch dental adhesives which have 10-MDP as a functional monomer used in class $\mathrm{V}$ cavity restoration. This in vitro study evaluates the dentin microtensile bond strength at the gingival margin of Class V cavities which were bonded with various 10-MDP contained dental adhesives for the resin composite restorations. For this purpose, 45 freshly extracted sound human upper premolars were prepared with water-cooled high-speed hand piece and cylindrical diamond bur [D8] at cemento-enamel junction on buccal surface of the teeth. The occlusal margin of cavity is located on enamel, and the gingival margin is located on cementum. The prepared teeth were randomly assigned into three groups of fifteen teeth. Microtensile bond strength ( $\mu \mathrm{TBS}$ ) was recorded, the failure patterns were classified, and the micromorphology of resin-dentine interfaces was evaluated. Results were analyzed by one-way ANOVA and Tukey's test $(\alpha=0.05)$. There were statistically significant higher $(p=0.019) \mu$ TBS value of SBU $(10.7664 \pm 4.2676$ $\mathrm{MPa})$ than CTS value $(6.4257 \pm 4.1786 \mathrm{MPa})$ but no statistically significant difference $(p>0.05)$ between SBU $(10.7664 \pm 4.2676 \mathrm{MPa})$ and CSE $(9.3257 \pm 3.6572 \mathrm{MPa})$, and between CSE $(9.3257 \pm 3.6572 \mathrm{MPa})$ and CTS (6.4257 $\pm 4.1786 \mathrm{MPa})$ were found. The adhesive failure between resin-dentin interfaces of three adhesive systems accounted for $75.33 \%$ of the total number of fractures and no cohesive failures were found in dentin. Considering the limitations of this in vitro study, some 2-step total etching dental adhesive which has 10-MDP as a functional monomer may be capable of producing higher microtensile bond strengths than 1-step self-etch dental adhesive which has 10-MDP as a functional monomer.
\end{abstract}

(C) 2017 The Author(s). Published by TAF Publishing.

\begin{abstract}
INTRODUCTION
The main objective of dental adhesive is to produce an adhesion between resin composite and tooth surface. Moreover, the suitable dental adhesive should be able to resist the mechanical force and shrinkage stress from polymerization procedure that cause the leakage within restoration's margin. In addition to poor seal ability of dental adhesive, the discoloration of restoration's margin could be found and the loss of restoration also finally occurs $[1,2,3]$.

The adhesion surface of dental adhesive is composed of two interfaces. First is the interface of adhesive and tooth surface, and second is the interface of adhesive and resin composite that develops from the polymerization process between oxygen inhibit layer
\end{abstract}

\footnotetext{
${ }^{*}$ Corresponding author: Suttinart Chomcheoy

†Email: chomcheoy@gmail.com
} 
of adhesive and resin composite. For the adhesion between adhesive and tooth surface (enamel and dentin), micromechanical adhesion is supposed to be the major mechanism [4]. This process of adhesion originates from replacing inorganic tooth material by resin monomers that become interlocked between tooth surface and adhesive layer $[5,6]$. Infiltration of resin monomer into etched tooth surface is the primary mechanism to gain micro-mechanical retention that appeared in microscope, called hybridization. Nakabayashi et al. [7] in the enamel, mechanical retention is created by infiltration of resin monomer into etched pits, whereas dentin obtains the retention from infiltration of resin monomer into exposed collagen layer and dentinal tubules. However, there are some selfetch adhesives using mild acidic property of monomer to obtain ionic bonding between acidic monomer and calcium in hydroxyapatite, [8] which could explain the good performance of this kind of self-etch adhesives in clinical usage $[9,10]$.

According to these bonding mechanisms, the primary bond efficiency may depend on micromechanical interlocking that happens after resin infiltration and polymerization happen in demineralized enamel and dentin. The chemical bonding could be initiated by using the specific monomer (functional monomer) that has chemical bond to hydroxyapatite. In addition, adequate co-polymerization between adhesive and resin composite could create a strong adhesion to the resin composite.

Many studies have reported a good performance of 10-MDP functional monomer [8, 9] and also has been used in recent dental adhesives of many manufacturers. The objective of this study is to evaluate the dentin microtensile bond strength of self-etch and total etching dental adhesives which have $10 \mathrm{MDP}$ as a functional monomer used in class V cavity restoration.

\section{LITERATURE REVIEWE}

For having a covalent bond between adhesive and resin composite from polymerization process, dental adhesives should be composed of similar kind of resin monomers to the resin composite. These kind of resin monomers act as a structural core providing physical and mechanical properties, such as strength. The monomers composed of dental adhesives could be divided into 2 groups; first is cross-linker monomer and second is functional monomer [11].

The cross-linker monomers have two polymerizable groups (vinyl-groups or $-\mathrm{C}=\mathrm{C}$-) or more [11]; di-methacrylates, such as Bis-GMA, UDMA, and TEGDMA are commonly used in dental adhesives and resin composite Figure 1 . These monomers directly supply mechanical strength to dental adhesive by creating densely cross-linked polymers [12]. The important characteristic of these monomers is hydrophobicity that prevents water absorption after curing [13]. A ranking in amount of water absorption could be sequenced: TEGDMA > Bis-GMA > UDMA [14]. Almost all adhesives composed of mixtures of these, and amount of each resin monomer will have a significant influence on the viscosity of the uncured adhesive [15], and mechanical properties of the cured adhesive [16, 17].

Bis-GMA is not only used in dental adhesives but also commonly used in resin composite. Due to the high molecular weight of Bis-GMA, it provides low polymerization shrinkage and rapid curing that result in good mechanical properties of the adhesives. In addition, UDMA is also commonly used in dental adhesives alone or in combination with Bis-GMA. Although UDMA has similar molecular weight to Bis-GMA, but UDMA exhibits lower viscosity properties than Bis-GMA [13]. 


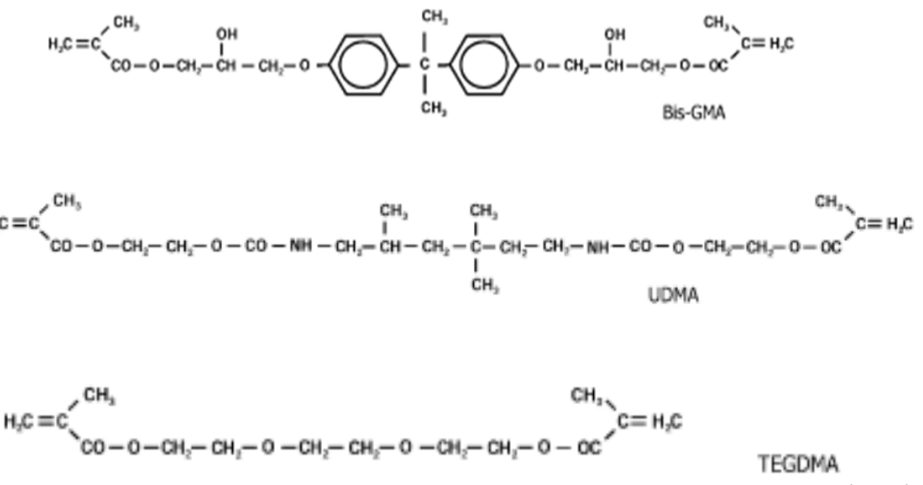

FIGURE 1. Chemical structure of Bis-GMA, UDMA, and TEGDMA

Due to lower viscosity properties of TEGDMA, it is usually used together with Bis-GMA and UDMA as diluents $[18,19]$. The higher flexibility of TEGDMA will compensate for the rigidity of Bis-GMA and admixture will result in higher conversion rate of resin [16]. In addition, TEGDMA increases tensile strength but reduces flexural strength of the cured resin [17].

Most functional monomers also have a particular chemical group that forms linear polymers upon curing, in contrast to cross-linkers that form cross-linking polymers [20]. The functional group in functional monomers usually exhibits hydrophilic properties. This group may have many purposes, such as enhancing wetting and demineralization of dentin that enhances bond strength of adhesives to dentin by their hydrophilic properties [21]. The functional groups generally used in commercial monomers are phosphate, carboxyl acid, and alcohol groups. Sulfonic acid, phosphate, phosphonate, and carboxyl group will dissociate to release protons in aqueous solutions, and will be able to react in acid-base reactions. Apart from "adhesion-promoting" or wetting effects, these proton-releasing functional groups may establish surface demineralization to a certain extent when applied to a sufficient concentration. A ranking on etching aggressiveness could be sequenced to acidity of these groups: sulfonic acid $>$ phosphonic $>$ phosphoric $>$ carboxylic acid $>$ alcohol $[22,23]$.

\section{0-MDP: 10-Methacryloyloxydecyl Dihydrogenphosphate}

10 -MDP is a monomer that was originally synthesized by Kuraray (Osaka, Japan) and hence patented by them Figure 2. It is mainly used as an etching monomer, due to the dihydrogenphosphate group, which can dissociate in water to form two protons [24]. Structurally, the long carbonyl chain renders this monomer quite hydrophobic. As a sequence, ethanol and acetone are most suitable for this monomer. Also, it is clear that 10-MDP will be relatively hydrolysis stable, as water will be kept at a distance. Yoshida et al. [8] showed that this monomer is capable of forming strong ionic bonds with calcium due to the low dissolution rate of resulting Ca-salt in its own solution.

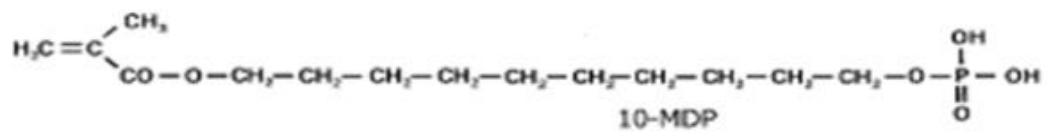

FIGURE 2. Chemical structure of 10-MDP 
The quality of the resin/tooth interface can be evaluated by the measurement of bond strength. This test can reveal the adhesion of the adhesive in clinical use. Bond strength can be evaluated by loading a specimen in shear or in tension until fracture is propagated. The specimen of conventional bond strength test has a large bonding area of about 7-12 $\mathrm{mm}^{2}$. This usually demonstrates cohesive failure in dentin $[25,26]$. Therefore, the conventional method does not supply the accurate results of the adhesive strength of the bonding agent. Furthermore, utilization of large surface area can produce the non-uniform interface area by containing air bubbles and phase separation, which could lead to unreliable results [27]. As a result, a new method of bond strength test, so called microtensile test, has been invented by Sano et al. [28]. This method produces higher bond strength than original methods. The primary adhesive failure at bonding interfaces, which are less than $2 \mathrm{~mm}^{2}$, could be detected [28]. The benefits of this method are to obtain more adhesive failures or fewer cohesive failures, to reveal higher interfacial bond strengths, to allow measurement of local bond strengths, to calculate means and variances for single teeth, to have ability to test bond strength of the irregular surfaces, to allow testing of very small area, and to utilize SEM examination to observe the failure mode of testing specimens. The disadvantages of this method are labor intensive or technically demanding and difficulty to measure bond strengths less than $5 \mathrm{MPa}$. Additionally, special equipment is required and samples are very small, which risk to rapidly dehydrate [29].

Class V cavities are composed of either enamel and dentin margins at occlusal and gingival margins accordingly. Dentin and enamel are different bonding substrates. Dentin has more organic materials and water than inorganic materials. Therefore, bond to dentin is harder than bond to enamel [30]. This in vitro study evaluates the dentin microtensile bond strength at the gingival margin of Class $V$ cavities which will be bonded with various 10-MDP contained dental adhesives for the resin composite restorations.

\section{Hypothesis}

H0: There was no significant difference of microtensile bond strength among various 10-MDP contained dental adhesives used for class $\mathrm{V}$ resin composite restoration.

H1: There was significant difference of microtensile bond strength among various 10-MDP contained dental adhesives used for class $\mathrm{V}$ resin composite restoration.

\section{Objective}

To compare the dentin microtensile bond strength of class $\mathrm{V}$ cavities which will be bonded by various $10-\mathrm{MDP}$ contained dental adhesives for class $\mathrm{V}$ resin composite restoration.

\section{MATERIALS AND METHODS}

\section{Materials}

- Scotchbond ${ }^{\mathrm{TM}}$ Etchant gel, 3M ESPE, Minnesota, USA 1 syringe.

- Single Bond Universal, 3M ESPE, Minnesota, USA 1 bottle.

- Clearfil SE Bond, Kuraray medical, Tokyo, Japan 1 set.

- Clearfil tri-S Bond, Kuraray medical, Tokyo, Japan 1 bottle.

- Estelite Sigma Quick [A3.5], Tokuyama Dental, Tokyo, Japan 3 tubes.

- $0.1 \%$ Thymol solution 3 . bottles

- Cyanoacrylate adhesive: Model Repair II blue, SANKIN, Tokyo, Japan 2 sets.

- Diamond disk, Intensive, Switzerland 10 pieces.

- Cylinder diamond bur [D8], Intensive, Switzerland 20 pieces.

- C16 diamond bur, Intensive, Switzerland 20 pieces. 
- Soflex Disk, 3M ESPE, Minnesota, USA 4 sets.

- Diamond Wafering Blade3"Dia.X0.015", Isocut ${ }^{\mathrm{TM}}$, Buehler, Lake Bluff, Illinois, USA.

- Epoxy resin, Epon815, Nissin, Tokyo, Japan.

- Silicon carbide paper: 600, 800, 1,000, 1,200 grit, Struers, Ballerup, Denmark. 100 pieces.

- Diamond paste, Dp-paste, Struers, Ballerup, Denmark.0.25,1,3,6 um [5g].

- Self-curing acrylic, Instant Tray Mix, Lang Dental Manufacturing Co., Inc. Wheeling, Illinois, USA.

- Gold Sputter Coater, SC-701AT Quick Coater, Elionix Ltd., Tokyo, Japan.

- Scanning electron microscope, Model JSM 5410 LV, JEOL Company, Tokyo, Japan.

- Universal testing machine, Instron Model 5566, Instron crop., Buckinghamshire, UK.

\section{Methods}

\section{Tooth specimen preparation}

Forty-five freshly extracted sound human premolars will be used in this study. The inclusion criteria are the teeth free from decay, cracks or restorations. After extraction, the teeth will be cleaned with pumice and stored in $0.1 \%$ thymol solution at $4^{0} \mathrm{C}$ before use. $\mathrm{V}$-shape cavities will be prepared with water-cooled high-speed hand piece and cylindrical diamond bur [D8] at cemento-enamel junction on buccal surface of the teeth. A bur will be used only for preparation of 4 cavities and then replaced by a new bur. The size of cavity will be $4.0 \times 2.6 \mathrm{~mm}$. The depth of cavity, determined with periodontal probe, will be approximately $2.0 \mathrm{~mm}$. The occlusal margin of cavity is located on enamel, and the gingival margin is located on cementum. The prepared teeth will be further randomly assigned into three groups of fifteen teeth and each will be restored with different materials and kept in normal saline solution until use.

\section{Restorative procedures}

Materials used in this study are demonstrated in Table 1. The prepared teeth of three groups of fifteen teeth will be restored according to the following conditions Figure 3.

Group 1: All cavity surfaces will be etched with $35 \%$ phosphoric acid [Scotchbond ${ }^{\mathrm{TM}}$ Etchant gel] for 15 seconds, rinsed for 10 seconds, and blot dried with a paper point. Bonding [Single Bond Universal] will be applied onto enamel and dentin. The bonded specimen will be gently dried for 5 seconds and light-cured for 10 seconds with a halogen curing unit [Elipar ${ }^{\mathrm{TM}}$ Trilight]. A resin composite [Estelite Sigma Quick, A3.5 shade] will be placed into the cavity with a bulk technique and cured for 40 seconds. Then, finishing and polishing with abrasive disks [Soflex Disk] in sequence will be performed immediately after polymerization.

Group 2: All cavity surfaces will be primed with Clearfil SE Bond [primer] for 20 seconds and gently dried for 5 seconds. Clearfil SE Bond [bonding] will be applied onto enamel and dentin. The bonded specimen will be gently dried for 5 seconds and lightcured for 10 seconds with a halogen curing unit [Elipar ${ }^{\mathrm{TM}}$ Trilight]. The resin composite [Estelite Sigma Quick, A3.5 shade] will be placed into the cavity with a bulk technique and cured for 40 seconds. Finishing and polishing will be performed as previously mentioned in group 1.

Group 3: All cavity surfaces will be applied with Clearfil tri-S Bond for 20 seconds onto enamel and dentin, dried with gentle air blow for 5 seconds, and light-cured for 10 seconds with a halogen curing unit [Elipar ${ }^{\mathrm{TM}}$ Trilight]. The resin composite [Estelite Sigma Quick, A3.5 shade] will be placed into the cavity with a bulk technique and cured for 40 seconds. Finishing and polishing will be performed previously mentioned in groups 1, 2 . 
TABLE 1. Materials used in this study

\begin{tabular}{|c|c|c|c|}
\hline Material & Type & Manufacturer & Composition \\
\hline $\begin{array}{l}\text { Single Bond } \\
\text { Universal Adhesive } \\
\text { (Group 1) }\end{array}$ & $\begin{array}{l}\text { One-bottle, } \\
\text { universal } \\
\text { adhesive }\end{array}$ & $\begin{array}{l}\text { 3M ESPE, } \\
\text { Seefeld, } \\
\text { Germany }\end{array}$ & $\begin{array}{l}\text { MDP, Dimethacrylate resins, } \\
\text { HEMA, Vitrebond }{ }^{\mathrm{TM}} \text { copolymer, } \\
\text { filler, ethanol, water, } \\
\text { silane, initiator }\end{array}$ \\
\hline $\begin{array}{l}\text { Scotchbond }{ }^{\mathrm{TM}} \\
\text { Etchant (Group 1) }\end{array}$ & Etching gel & $\begin{array}{l}\text { 3M ESPE, } \\
\text { Seefeld, } \\
\text { Germany }\end{array}$ & $\begin{array}{l}\text { 35\% Phosphoric } \\
\text { acid }\end{array}$ \\
\hline $\begin{array}{l}\text { Clearfil SE } \\
\text { Bond (Group 2) }\end{array}$ & $\begin{array}{l}\text { Two-steps, } \\
\text { self-etch adhesive }\end{array}$ & $\begin{array}{l}\text { Kuraray Medical, } \\
\text { Tokyo, Japan }\end{array}$ & $\begin{array}{l}\text { Primer: MDP, HEMA, } \\
\text { hydrophilic dimethacrylate, photo- } \\
\text { initiator, water Bond: } \\
\text { MDP, HEMA, Bis-GMA, hydrophobic } \\
\text { dimethacrylate, photo-initiator, } \\
\text { silanated colloidal silica }\end{array}$ \\
\hline $\begin{array}{l}\text { Clearfil Tri-S } \\
\text { Bond (Group 3) }\end{array}$ & $\begin{array}{l}\text { One-step, } \\
\text { self-etch adhesive }\end{array}$ & $\begin{array}{l}\text { Kuraray Medical, } \\
\text { Tokyo, Japan }\end{array}$ & $\begin{array}{l}\text { MDP, HEMA, initiator, } \\
\text { ethanol, water, stabilizer, } \\
\text { filler, hydrophobic dimethacrylate }\end{array}$ \\
\hline $\begin{array}{l}\text { Esthelite sigma } \\
\text { quick (Group 1,2,3) }\end{array}$ & $\begin{array}{l}\text { Nano-hybrid } \\
\text { resin composite }\end{array}$ & $\begin{array}{l}\text { Tokuyama Dental, } \\
\text { Japan }\end{array}$ & $\begin{array}{l}\text { Bis-GMA,UDMA, hydrophobic } \\
\text { dimethacrylate, photo-initiator, } \\
\text { silanated colloidal silica }\end{array}$ \\
\hline
\end{tabular}

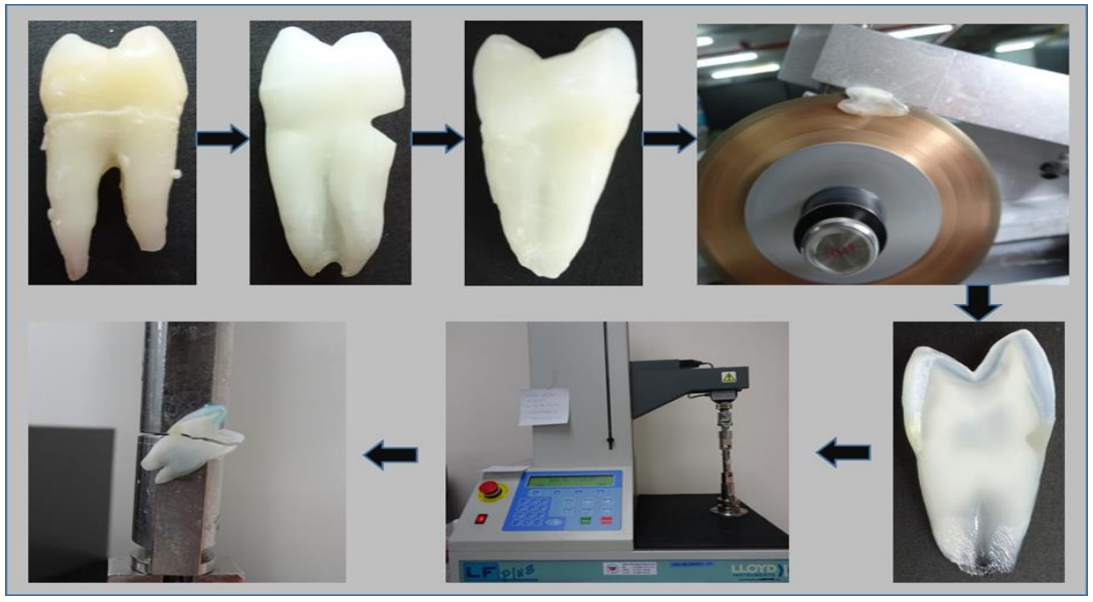

FIGURE 3 . Flowchart of the specimen preparation

After complete restore, each group of fifteen teeth will be divided into two subgroups of thirteen teeth and two teeth; first subgroup of thirteen teeth will be subjected to microtensile bond strength test, and second subgroup of two teeth will be subjected to micromorphology evaluation.

\section{Evaluation of microtensile bond strength}

The specimens for microtensile test will be sectioned bucco-lingually into 2 slabs of 0.7 $\mathrm{mm}$. thick using low-speed diamond saw. The slabs of specimens will be trimmed at gingival wall into dumbbell shape by a super-fine point bur [C16] under water coolant. The cross-sectioned area at resin-dentin interface will be approximately $1.0 \mathrm{~mm}^{2}$. The 
trimmed specimens will be attached to a testing apparatus [Bencor-T Multi testing apparatus] with a cyanoacrylate adhesive [Model Repair II Blue] on a universal testing machine [Instron Model 5566]. The tensile forces will be applied at a cross-head speed of 1 $\mathrm{mm} / \mathrm{min}$. The fracture strength will be calculated from maximal force with the attached computer and converted into MPa.

\section{Evaluation of mode of fracture}

After microtensile bond strength evaluation, the couple of the specimens will be observed for failure mode under a scanning electron microscope [JSM5410LV] at 1,500 and 3,500 magnifications.

\section{Evaluation of micromorphology of resin-dentine interfaces}

The two teeth selected from each group will be sectioned bucco-lingually with a low-speed saw with diamond wafering blade and kept in $10 \%$ buffer formalin for 24 hours. Specimens will be then rinsed with distilled water and embedded in epoxy resin in PVC ring. After 24 hours, the embedded section will be polished with wet silicon carbide paper of decreasing abrasiveness $[600,800,1000,1200$ grit] and diamond paste down to $0.25 \mathrm{um}$ grain. The polished specimens will be immersed in $40 \%$ phosphoric acid for 30 seconds, rinsed under distilled water for 20 seconds, and will be dried by air blow. The dried specimens will be sputter-coated and observed resin-dentine interface under a scanning electron microscope [JSM5410LV] at 1,500 and 3,500 magnifications.

\section{Statistical analysis}

- One-way ANOVA.

- Duncan multiple comparison.

\section{RESULTS}

\section{Evaluation of Microtensile Bond Strength}

The mean microtensile bond strength values and mode of fractured are presented in Table 2. The ANOVA test at the 0.05 significance level demonstrated a difference between the groups tested $(d f=2, F=4.186, p<0.05$ ) that was further compared with the Tukey HSD test to show significant differences between SBU and CTS $(p=0.019)$. There was no significant difference between SBU and CSE ( $p=0.617)$, and between CSE and CTS ( $p=0.153$ ). The highest mean microtensile bond strength value $(10.7664 \pm 4.2676 \mathrm{MPa})$ was found in group bonded with Single Bond Universal and significantly higher than in the Clearfil Tri-s Bond group (6.4257 $\pm 4.1786 \mathrm{MPa}$ ). The mean microtensile bond strength value of Clearfil SE Bond group $(9.3257 \pm 3.6572 \mathrm{MPa})$ was higher but not significantly different from the value of Clearfil Tri-S Bond group. Same letters connect groups that are not statistically and significantly different $(p<0.05)$.

TABLE 2 . Mean microtensile bond strength values (MPa) of 10-MDP contained dental adhesives

\begin{tabular}{ll}
\hline \hline Adhesive System & Mean $\pm \boldsymbol{S D}$ \\
\hline Single bond universal & $10.7664 \pm 4.2676^{\mathrm{a}}$ \\
Clearfil SE bond & $9.3257 \pm 3.6572^{\mathrm{ab}}$ \\
Clearfil Tri-S bond & $6.4257 \pm 4.1786^{\mathrm{b}}$ \\
\hline \hline
\end{tabular}




\section{Evaluation of Mode of Fracture}

The failure modes of three adhesive systems are presented in Table 3, the adhesive fractures accounted for $75.33 \%$ of the total number of fractures, and no cohesive failures were found in dentin.

TABLE 3 . Percentage of
failure mode
(Aadhesive,
M-mixed) of
10-MDP conta-
ined dental
adhesives

\section{Evaluation of Micromorphology of Resin-Dentine Interfaces}

The SEM photomicrographs of each experimental group are shown in Figure 4. All systems were able to penetrate the demineralized dentin that created hybrid layer and resin tags. SBU demonstrates the longest resin tags, followed by the length of CSE and CTS, respectively.

Scanning electron micrographs illustrating interfacial morphology of three 10-MDP contained dental adhesives. Hybrid layer is represented by $H$ and resin tag is represented by $T$.
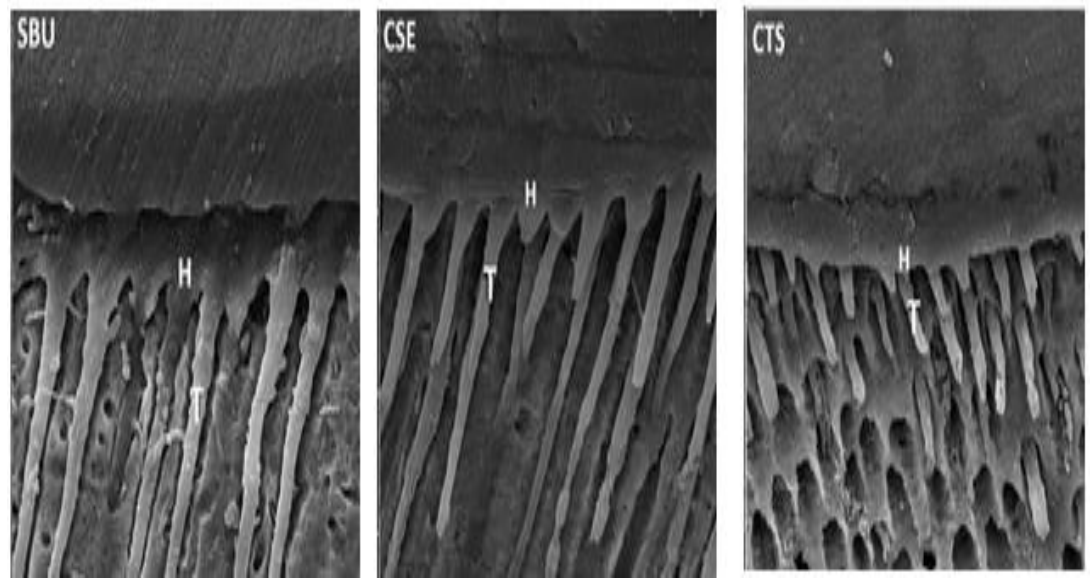

FIGURE 4. Scanning electron micrographs

\section{DISCUSSION}

The method utilized for bond strength testing was the microtensile bond strength test that was reported to be suitable for the evaluation of interfacial bond strengths on areas below $1 \mathrm{~mm}^{2}$ [31]. However, the trimming technique might have adopted the number of prema- 
ture failures during specimen preparation [32] that caused the microtensile bond strength values in this study to be lower than some previous studies [25, 26, 27, 28, 29, 30].

SBU, CSE, and CTS are all 10-MDP-based adhesives that showed the low $\mathrm{pH}$ values and also enabled simultaneous demineralization and monomer infiltration. Moreover, 10-MDP is a recognized monomer able to chemically interact with tooth minerals [33], improving the long-term stability of the adhesion formed. Espacially for SBU, it is also comprised of a polyalkenoic acid copolymer (Vitrebond ${ }^{\mathrm{TM}}$ copolymer), which, according to the manufacturer, provides satisfactory bonding to dentin under moist or dry conditions [33]. Thease reasons might cause the mean microtensile bond strength value of SBU to be the hightest and significantly higher than in CTS group.

The lowest mean microtensile bond strength value was found in group bonded with CTS that might be explained by the 'all-in-one' or 'simplified' characteristic of this kind of adhesive. Lower bond strengths of simplified adhesives are usually explained as a consequence of increased hydrophilicity due to joining primer and adhesive in one bottle [34]. The increased concentration of hydrophilic resin monomers in simplified all-in-one adhesives may cause the remnants of water that remained or trapped in the adhesive layer. Studies have shown that water may exist in the adhesive layer as free, within the collagen matrix, or bound, if it forms hydrogen bonds with hydrophilic resin monomers [35, 36]. In areas where water remains, polymerization may be incomplete. Also, these areas represent porous regions which may facilitate hydrolytic degradation of adhesive interface. Therefore, it is very important that water and solvents are completely removed, following the adhesive application. In order to accomplish this goal, manufacturers recommend air drying of various durations and intensities. Yiu et al. have investigated the percentage of water and solvent that remains in the adhesive after drying, simulating clinical conditions [37]. It was reported that the percentage of remaining solvent increases with the increasing hydrophilicity of the adhesive.

More studies about the correlation between microtensile bond strength and the length of resin tag are necessary to conclude this relationship. In this study, SEM photomicrographs of SBU demonstrate the longest lehgth of resin tags, followed by the length of CSE and CTS, respectively that correlate with mean microtensile bond strength value of each group. Meanwhile, the study conducted by Garcia de Oliveira et al. [39] concludes that the bond strength of dental adhesive to dentin is not dependent on the hybrid layer thickness and length of resin tags.

\section{CONCLUSION}

Considering the limitations of this in vitro study, some 2-step total etching dental adhesive which has 10-MDP as a functional monomer may be capable of producing higher microtensile bond strengths than 1-step self-etch dental adhesive which has 10-MDP as a functional monomer.

\section{REFERENCES}

1. Gaengler P, Hoyer I, Montag R, Gaebler P. Micromorphological evaluation of posterior composite restorations-a 10-year report. Journal of Oral Rehabilitation. 2004; 31(10): 991-1000. DOI: 10.1111/j.1365-2842.2004.01329.x

2. Opdam NJ, Loomans BA, Roeters FJ, Bronkhorst EM. Five-year clinical performance of posterior resin composite restorations placed by dental students. Journal of Dentistry. 2004; 32(5): 379-383. DOI: 10.1016/j.jdent.2004.02.005 
3. Dokhan T, Shebani N, Zaet A. Relationship of bolton's ratios and tooth-size discrepancy. Journal of Advances in Health and Medical Sciences. 2016; 2(1): 34-41. DOI: 10.20474/jahms-2.1.5

4. Van Meerbeek B, Vargas M, Inoue S, Yoshida Y, Peumans M, Lambrechts P, Vanherle G. Adhesives and cements to promote preservation dentistry. Operative Dentistry. 2001; 26: 119-44. DOI: 10.1016/s0300-5712(96)00070-x

5. Asmussen E, Hansen EK, Peutzfeldt A. Influence of the solubility parameter of intermediary resin on the effectiveness of the Gluma bonding system. Journal of Dental Research. 1991; 70(9): 1290-1293.

DOI: $10.1177 / 00220345910700091101$

6. Van Meerbeek B, De Munck J, Yoshida Y, Inoue S, Vargas M, Vijay P, Van Landuyt K, Lambrechts P, Vanherle G. Adhesion to enamel and dentin: Current status and future challenges. Operative Dentistry-University Of Washington. 2003; 28(3): 215-235.

7. Nakabayashi N, Kojima K, Masuhara E. The promotion of adhesion by the infiltration of monomers into tooth substrates. Journal of Biomedical Materials Research Part A. 1982; 16(3): 265-273. D0I: 10.1002/jbm.820160307

8. Yoshida Y, Nagakane K, Fukuda R, Nakayama Y, Okazaki M, Shintani H, Inoue S, Tagawa Y, Suzuki K, De Munck J, Van Meerbeek B. Comparative study on adhesive performance of functional monomers. Journal of Dental Research. 2004; 83(6): 454-458. DOI: $10.1177 / 154405910408300604$

9. Peumans M, Kanumilli P, De Munck J, Van Landuyt K, Lambrechts P, Van Meerbeek B. Clinical effectiveness of contemporary adhesives: A systematic review of current clinical trials. Dental Materials. 2005; 21(9): 864-881.

DOI: $10.1016 /$ j.dental.2005.02.003

10. La Roche LAT, Herrmann A, Cezar C, Larbig A, Leicher L, De Wilde MS, De Wilde RL, Prophylaxis of peritoneal adhesions: Practical issues to consider when using antiadhesion agents. International Journal of Health and Medical Sciences. 2017; 3(1): 01-05. DOI: 10.20469/ijhms.3.30001-1

11. Coessens V, Pintauer T, Matyjaszewski K. Functional polymers by atom transfer radical polymerization. Progress in Polymer Science. 2001; 26(3): 337-377. D0I: 10.1016/s0079-6700(01)00003-x

12. Sideridou I, Tserki V, Papanastasiou G. Effect of chemical structure on degree of conversion in light-cured dimethacrylatebased dental resins. Biomaterials. 2002; 23(8): 1819-1829. DOI: 10.1016/s0142-9612(01)00308-8

13. Peutzfeldt A. Resin composites in dentistry: The monomer systems. European Journal of Oral Sciences. 1997; 105(2): 97-116. DOI: 10.1111/j.1600-0722.1997.tb00188.x

14. Sideridou I, Tserki V, Papanastasiou G. Study of water sorption, solubility and modulus of elasticity of light-cured dimethacrylate-based dental resins. Biomaterials. 2003; 24(4): 655-665. DOI: 10.1016/s0142-9612(02)00380-0

15. Silikas N, Watts DC. Rheology of urethane dimethacrylate and diluent formulations. Dental Materials. 1999; 15(4): 257-261. DOI: 10.1016/s0109-5641(99)00043-3

16. Asmussen E, Peutzfeldt A. Influence of selected components on crosslink density in polymer structures. European Journal of Oral Sciences. 2001; 109(4): 282-285. DOI: 10.1034/j.1600-0722.2001.00057.x

17. Asmussen E, Peutzfeldt A. Influence of UEDMA, BisGMA and TEGDMA on selected mechanical properties of experimental resin composites. Dental Materials. 1998; 14(1): 51-56. DOI: 10.1016/s0109-5641(98)00009-8

18. Labella R, Davy KW, Lambrechts P, Meerbeek BV, Vanherle G. Monomethacrylate co-monomers for dental resins. European Journal of Oral Sciences. 1998; 106(3): 816-824. DOI: 10.1046/j.0909-8836.1998.eos106308.x

19. Lu H, Stansbury JW, Nie J, Berchtold KA, Bowman CN. Development of highly reactive mono-(meth) acrylates as reactive diluents for dimethacrylate-based dental resin systems. Biomaterials. 2005; 26(12): 1329-1336.

DOI: $10.1016 /$ j.biomaterials.2004.04.041

20. Sheldon RP. Composite polymeric materials. New York, NY: Applied Science Publishers; 1982.

21. Watanabe I, Nakabayashi N, Pashley DH. Bonding to ground dentin by a phenyl-P self-etching primer. Journal of Dental Research. 1994; 73(6): 1212-1220. DOI: 10.1177/00220345940730061301

22. Nishiyama N, Suzuki K, Yoshida H, Teshima H, Nemoto K. Hydrolytic stability of methacrylamide in acidic aqueous solution. Biomaterials. 2004; 25(6): 965-969. DOI: 10.1016/s0142-9612(03)00616-1 
23. Moszner N, Salz U, Zimmermann J. Chemical aspects of self-etching enamel-dentin adhesives: A systematic review. Dental Materials. 2005; 21(10): 895-910. DOI: 10.1016/j.dental.2005.05.001

24. Hayakawa T, Kikutake K, Nemoto K. Influence of self-etching primer treatment on the adhesion of resin composite to polished dentin and enamel. Dental Materials. 1998; 14(2): 99-105. DOI: 10.1016/s0109-5641(98)00015-3

25. Perinka L, Sano H, Hosoda H. Dentin thickness, hardness, and Ca-concentration vs bond strength of dentin adhesives. Dental Materials. 1992; 8(4): 229-233. D0I: 10.1016/0109-5641(92)90090-y

26. Erickson RL, Glasspoole EA, Retief DH. Effect of air thinning on bond strength measurements. Journal of Dental Research. 1989;68 (3): 489-490. DOI: 10.1177/00220345890680031101

27. Versluis A, Tantbirojn D, Douglas WH. Why do shear bond tests pull out dentin? Journal of Dental Research. 1997; 76(6): 1298-1307. DOI: 10.1177/00220345970760061001

28. Sano H, Shono T, Sonoda H, Takatsu T, Ciucchi B, Carvalho R, Pashley DH. Relationship between surface area for adhesion and tensile bond strength—evaluation of a micro-tensile bond test. Dental Materials. 1994; 10(4): 236-240.

DOI: $10.1016 / 0109-5641(94) 90067-1$

29. Pashley DH, Sano H, Ciucchi B, Yoshiyama M, Carvalho RM. Adhesion testing of dentin bonding agents: A review. Dental Materials. 1995; 11(2): 117-125. DOI: 10.1016/0109-5641(95)80046-8

30. Ben-Amar A, Pilo R, Shapinko E, Lewinstein I. A microleakage study of single-bottle adhesives applied to enamel and cementum and aged by both occlusal loading and thermocycling. Quintessence International. 2005; 36(3): 292-297. DOI: $10.1016 /$ j.prosdent.2005.05.010

31. Elkassas D, Taher HA, Elsahn N, Hafez R, El-Badrawy W. Effect of the number of applications of acetone-based adhesives on microtensile bond strength and the hybrid layer. Operative Dentistry. 2009; 34(6): 688-696. D0I: 10.2341/08-089-1

32. Sadek FT, Monticelli F, Goracci C, Tay FR, Cardoso PE, Ferrari M. Bond strength performance of different resin composites used as core materials around fiber posts. Dental Materials. 2007; 23(1): 95-99. D0I: 10.1016/j.dental.2005.12.005

33. Van Meerbeek B, Yoshihara K, Yoshida Y, Mine AJ, De Munck J, Van Landuyt KL. State of the art of self-etch adhesives. Dental materials. 2011; 27(1): 17-28. D0I: 10.1016/j.dental.2010.10.023

34. Espe M. Scotchbond ${ }^{T M}$ universal adhesive; 2014. Available from: goo.gl/JSBCAg

35. Magni E, Mazzitelli C, Papacchini F, Radovic I, Goracci C, Coniglio I, Ferrari M. Adhesion between fiber posts and resin luting agents: A microtensile bond strength test and an SEM investigation following different treatments of the post surface. Journal of Adhesive Dentistry. 2007; 9(2): 195-202.

36. Tay FR, Pashley DH, Suh BI, Carvalho RM, Itthagarun A. Single-step adhesives are permeable membranes. Journal of Dentistry. 2002; 30(7-8): 371-382. D0I: 10.1016/s0300-5712(02)00064-7

37. Suh BI, Feng L, Pashley DH, Tay FR. Factors contributing to the incompatibility between simplified-step adhesives and chemically-cured or dual-cured composites. Part III effect of acidic resin monomers. Journal of Adhesive Dentistry. 2003; 5(4): 23-45.

38. Yiu CK, Pashley EL, Hiraishi N, King NM, Goracci C, Ferrari M, Carvalho RM, Pashley DH, Tay FR. Solvent and water retention in dental adhesive blends after evaporation. Biomaterials. 2005; 26(34): 6863-6872.

DOI: $10.1016 /$ j.biomaterials.2005.05.011

39. Garcia de Oliveira F, Bruniera Anchieta R, Rahal V, Sversut de Alexandre R, Silveira Machado L, Marçal Mazza Sundefeld ML, Giannini M, Sundfeld RH. Correlation of the hybrid layer thickness and resin tags length with the bond strength of a self-etching adhesive system. Acta Odontológica Latinoamericana. 2009; 22(3): 177-181.

DOI: $10.1016 /$ j.jdent.2005.01.011

— This article does not have any appendix. - 